\title{
Biological potentials for a family of disintegrin and metalloproteinase (ADAMDEC)-1 in mouse normal pregnancy
}

\author{
Nobue KUNIYOSHI'), Hiroyuki IMAI'), Yasuo KISO'), Orie NAGAOKA ${ }^{2)}$ and \\ Ken Takeshi KUSAKABE ${ }^{1,2) *}$ \\ 1)Laboratory of Basic Veterinary Science, The United Graduate School of Veterinary Science, Yamaguchi University, \\ Yamaguchi 753-8515, Japan \\ 2)Laboratory of Veterinary Anatomy, Joint Faculty of Veterinary Medicine, Yamaguchi University, \\ Yamaguchi 753-8515, Japan
}

J. Vet. Med. Sci.

83(3): 512-521, 2021

doi: $10.1292 /$ jvms.20-0570

Received: 28 September 2020 Accepted: 19 January 2021 Advanced Epub:

19 February 2021

\begin{abstract}
Our previous research has indicated local expression of ADAMDEC-1, a family of disintegrin and metalloproteinase, was confirmed in the mouse placentas and enhancement was found in the sites for spontaneous abortion. Present study was aimed to identify biological effects of ADAMDEC-1 in pregnancy process. Syngeneic pairs of C57BL/6J mice and heterogenic mating pairs of $C B A / J$ and $D B A / 2$ mice were used. Pregnant mice were treated with recombinant ADAMDEC-1 protein. Vasculogenesis effects was evaluated using the Matrigel plugs including vascular endothelial growth factor singularity or combination with ADAMDEC-1. ADAMDEC-1 single effects were evaluated by tubal formation and proliferation assays using HuEht-1 endothelial cells. Expression of ADAMDEC-1 was not exactly corresponded with the time periods for miscarriage initiation. ADAMDEC-1 was distributed in normal placentas and fetuses, especially at extraembryonic ectoderm, decidua cells, uterine natural killer (uNK) cells in decidua, trophoblasts in labyrinthine zone, and hematopoietic cells in umbilical blood and fetal liver. ADAMDEC-1 treatment did not affect reproductive performances, while it elevated uNK cell recruitment in placenta and enlarged lumen sizes of the intraplacental vessels. In vitro analysis also indicated ADAMDEC-1 promoting effect on tubal formation and cell length of HuEht-1. qPCR analysis showed that ADAMDEC-1 modified placental gene expression especially for linkage of actin filament rearrangement. Our findings suggested that ADAMDEC-1 is correlated on cell shape, stability, and movement via modification of actin cytoskeleton. ADMADEC-1 suspected to regulate cellular activity of endothelial cells, trophoblasts, and uNK cells and may support normal developing of mouse placentas.
\end{abstract}

KEY WORDS: a disintegrin and metalloprotease-like decysin-1, epithelial cell, trophoblast, uterine NK cell

In the placentas during pregnant periods, various types of physiological factors mobilize and contribute to pregnancy success. We have tried comprehensive genetic analysis on mouse placental tissues in normal pregnancy and compared with the tissue from spontaneous abortion by the DNA microarray [16]. Specific gene fluctuations were found on the immunological, hormonal and hematological factors that were suggested to be closely correlated to spontaneous abortion occurrence. From the candidates of immunological factors, the complement factors have been proven to participate in the process of miscarriage induction and to possess immunosuppressive effects available for normal pregnancy [25-27]. In the present study, another fluctuated factor named as a disintegrin and metalloprotease-like decysin (ADAMDEC)-1 was targeted, because its biological effects on pregnant physiology have been established yet.

ADAMDEC-1 belongs to a family protein of disintegrin and metalloproteinase (ADAM). ADAM family is composed of more than 25 kinds of related proteins and the common frameworks are formed by proteinase, disintegrin, and cell signaling domains [6]. Specific substrates for the proteinase domain have been examined on several types of ADAM proteins and they can bring physiological effects. ADAM-17 can cleave precursor of tumor necrosis factor (TNF)- $\alpha$ and effect as a "TNF- $\alpha$ converting enzyme". ADAM-10 can digest the ectodomain of Notch and suppress Notch ligand binding, which is called as "ectodomain shedding" effects [6]. Regarding ADAMDEC-1, the specific catalytic substrate and physiological effects remains unclear.

*Correspondence to: Kusakabe, K.-T.: kusakabe@yamaguchi-u.ac.jp (Supplementary material: refer to PMC https://www.ncbi.nlm.nih.gov/pmc/journals/2350/) (O2021 The Japanese Society of Veterinary Science

This is an open-access article distributed under the terms of the Creative Commons Attribution Non-Commercial No Derivatives (by-nc-nd) License. (CC-BY-NC-ND 4.0: https://creativecommons.org/licenses/by-nc-nd/4.0/) 
ADAMDEC-1 in mouse pregnant uteri has been reported as an induced gene by prolactin receptor and/or progesterone-derived signaling pathways [4]. This report clarified ADAMDEC-1 placental distribution during days 5.5-12.5 of pregnancy and suggested a vital role for angiogenesis or vascularization during endometrium decidualization and embryo growth. However, this report contradicted our findings, i.e., high intense expression for ADAMDEC-1 was found at the spontaneously aborted placenta at day 14 of pregnancy [16]. ADAMDEC-1 is also pointed out as a candidate for arterial sclerosis histogenesis in human carotid artery [24]. Fundamental knowledge for ADAMDEC-1 is insufficient to discuss its effects on reproduction. Firstly, we examined mRNA and protein appearances of ADAMDEC-1 in normal and miscarriage placenta and forwarded experiments were designed to discuss the responsibility for ADAMDEC-1 in mouse reproduction.

\section{MATERIALS AND METHODS}

\section{Laboratory mice}

C57BL/6J (B6, Japan SLC, Hamamatsu, Japan), CBA/J (CBA, Charles River, Yokohama, Japan), DBA/2 strains (DBA, Japan CLEA, Tokyo, Japan) were used at the 8-10 weeks of age. All mice were bred with free access to water and food under the controlled condition of temperature and humidity $\left(22 \pm 2{ }^{\circ} \mathrm{C}\right.$ and $\left.50 \pm 10 \%\right)$ and the 12:12-hr light-dark cycle. After 1-week acclimation, B6 mice were paired in syngeneic at evening, and the vaginal plug formation at the next morning was defined at day 0.5 of pregnancy (D0.5). Mice were euthanized by cervical dislocation after deep inherent anesthesia using isoflurane. Pregnant uteri were collected and treated with an appropriate fixative or rapid freezing corresponding to the following experiments.

Miscarriage placentas were efficiently collected from the allogeneic pairs between female CBA (type of major histocompatibility antigen; $\left.\mathrm{H}-2^{\mathrm{k}}\right)$ and male DBA $\left(\mathrm{H}-2^{\mathrm{d}}\right)$ mice, which combination is well known to cause spontaneous abortion frequently [26]. Reproductive performances from those mating pairs were shown in Supplementary Table 1. Normal and miscarriage implantation sites were judged from stereomicroscope observation and microdissection to confirm the size, discolorization and degeneration degree (Supplementary Fig. 1). All animals were received humane care along the NIH guide for the Care and Use of Laboratory Animals. The experimental designs using laboratory animals were approved by the Ethical Committee on Animal Experimentation at Yamaguchi University (Approval number: 271).

\section{Treatments to animals}

Recombinant ADAMDEC-1 protein (Novoprotein, Summit, NJ, USA) or same volume of vehicle (control) were intraperitoneally injected to pregnant B6 mice. ADAMDEC-1 injection was adjusted to $100 \mathrm{ng} / \mathrm{ml}$ in total blood volume and the dose setting was referred from the present results of cell culture mentioned later. Total blood volume of mouse was configured as $1 \mathrm{ml}$ per $10 \mathrm{~g}$ body weight. From the present results of intrinsic expression pattern for ADAMDEC-1 in mouse placentas, ADAMDEC-1 was administered at D11.5, 12.5 and 13.5. Following treatments, pregnant uteri were collected at D14.5 and then macroscopic observation, weight measurement, and specimen preparation for next analyses were conducted on each implantation sites.

\section{Matrigel transplant assay}

This analysis was conducted to evaluate ADAMDEC-1on vasculogenesis and vasoactive effects. Matrigel (basement membrane matrix growth factor reduced, Corning, Bedford, MA, USA) was thawed on ice and mixed with $5 \mathrm{ng} / \mathrm{ml}$ of recombinant vascular endothelial growth factor (VEGF, Wako, Osaka, Japan). Prepared Matrigel was injected $(0.5 \mathrm{ml})$ to the axillary cavity of non-pregnant female B6 mice under inhalant anesthesia with isoflurane. Liquefied Matrigel become polymerized around $25^{\circ} \mathrm{C}$ condition. After 5 days from Matrigel injection, $100 \mathrm{ng} / \mathrm{ml}$ of ADAMDEC-1 or same volume of saline vehicle was additionally injected to the same side of axillary cavity. Dose settings for VEGF and ADAMDEC-1 addition were referred from the previous report [2] and our cell culture study mentioned below, respectively. After 10 days from the final treatment, solidified Matrigel transplants were collected from sacrificed mice (Fig. 3A) and fixed with 2\% paraformaldehyde (PFA). Cryostat sections were prepared from the specimens embedded by O.C.T. compound (Sakura Finetek Japan, Tokyo, Japan). Following Hematoxylin-Eosin (H-E) staining, measurements were conducted regarding the number of vessels invaded into the Matrigel transplants, the vessel size, and minimum length of the inner diameter.

\section{Histological studies}

H-E staining were performed on the dehydrated paraffin sections that prepared from the specimens fixed with $10 \%$ neutralbuffered formalin. Immunohistochemical staining were conducted on the dehydrated paraffin sections following autoclave treatment with $20 \mathrm{mM}$ glycine-HCl buffer (pH 3.0). Sections were treated with 10\% normal rabbit serum (Dako Japan, Tokyo, Japan) and reacted with goat polyclonal anti-ADAMDEC-1 antibody (Santa Cruz Biotech, Santa Cruz, CA, USA) and Histofine MAX-PO (G) reagent (including second antibody, Nichirei Biosciences, Tokyo, Japan). Other dehydrated sections were treated with $1 \%$ bovine serum albumin and then with biotinylated Dolichos Biflorus Agglutinin (DBA lectin, Vector Lab, Burlingame, CA, USA) or biotinylated Bandeirasea simplicifolia Isolectin (BSI)-B4 (Sigma-Aldrich, St. Louis, MO, USA). DBA lectin has been used as a specific marker for uterine natural killer (uNK) cells [23] and BSI-B4 was used for the specific detection for vascular endothelium. Sections were then reacted with $\mathrm{ABC}$ reagent (Vector Lab).

The peroxidase reactivity carried by secondary antibodies or ABC reagent was visualized by Peroxidase Stain DAB Kit (Nacalai Tesque, Kyoto, Japan). Counterstaining was conducted briefly with the hematoxylin. Spiral arteries and fetal capillaries were observed respectively at decidua basalis and placental labyrinthine areas and those of the size were measured using BZ-II analyzer 
(Keyence, Osaka, Japan) on short diameter of the inner lumen size. uNK cells were distinguished and counted according to Paffaro's morphological criteria [23], along the type I (immature), II (developed), III (matured), and IV (degenerated).

\section{Quantitative PCR (qPCR) analysis}

Total RNA was extracted from the frozen fresh specimens using ISOGEN2 Regent for RNA Extraction kit (Nippon Gene, Tokyo, Japan). cDNA was synthetized by reverse transcription reaction using ReverTra Ace qPCR kit (TOYOBO, Osaka, Japan). Primer sequences were designed using Primer3Plus software (www.primer3plus.com) and summarized in Supplementary Table 2. Real-time PCR analyses were performed using KAPA SYBR FAST kit (Nippon Genetics, Tokyo, Japan) and StepOne Plus realtime PCR System (Thermo Fisher Scientific, Waltham, MS, USA). Results were corrected with the amount of the gapdh expression and evaluated by $\Delta \Delta \mathrm{Ct}$ relative quantification method.

\section{Cell culture}

In vitro evaluations for ADAMDEC-1 effects were conducted on HUEhT-1 epithelial cell line (No. JCRB1458 in JCRB Cell Bank from National Institutes of Biomedical Innovation, Health and Nutrition, Osaka, Japan). HUEhT-1 has been established from human umbilical vein endothelial cell (HUVEC) line and operated genetical modification for constant expression of human telomerase reverse transcriptase. Accordingly, HUEhT-1 cells acquired immortal in life span bearing normal morphology and same growth level with HUVEC [2]. Cells were grown in the condition medium [10\% fetal bovine serum (Gibco, Grand Island, NY, USA), $10 \mathrm{mM}$ L-glutamate, $5 \mu \mathrm{g} / \mathrm{ml}$ heparin (Sigma-Aldrich), $0.03 \mathrm{~g} / 1$ endothelial cell growth supplement (Corning) in MCDB131 medium (Gibco)] on the collagen-coated dish in $5 \% \mathrm{CO}_{2}$ and $37^{\circ} \mathrm{C}$.

Confluently grown HUEhT-1 cells were dispersed to Matrigel coated 25 -well plates $\left(1.5 \times 10^{5}\right.$ cells/well $)$ and then cultured in the condition medium added with $0-1,000 \mathrm{ng} / \mathrm{ml}$ of recombinant ADAMDEC-1 (Novoprotein). After 18-20 hr, most of cells showed dynamic morphological change i.e., mutual cellular connection and tube-like structure formation (Fig. 2A, 2B). Cells were fixed with 2\% PFA and the lumen area in each tube was measured using cellSens software (OLYMPUS, Tokyo, Japan).

Other confluent HUEhT-1 cells were dispersed to 96 -well plate $\left(1.5 \times 10^{3}\right.$ cells/well $)$ and cultured with $0 \mathrm{or} 100 \mathrm{ng} / \mathrm{ml}$ of ADAMDEC-1. Setting for ADAMDEC-1 dose was referred by the tube-formation results. After 24 and 48 hr, cell growth activity was evaluated using Cell Counting Kit-8 (CCK8; Dojindo, Kumamoto, Japan). Absorbance at $450 \mathrm{~nm}$ was measured using DTX multimode detector (Beckman Coulter, Fullerton, CA, USA).

\section{Statistical analysis}

Data were collected from more than three specimens per group and presented as means \pm standard error (SE). Statistical comparisons between two groups were performed by the methods of Student's $t$-test or Mann-Whitney test following the evaluation with variance equality $F$-test. The probability value $<0.05$ was considered as significant.

\section{RESULTS}

\section{Expression of ADAMDEC-1 in mouse pregnancy periods}

qPCR analysis could detect the ADAMDEC-1 expression in the placentas of D6.5 of pregnancy (Fig. 1A). The expression level increased more than double at D10.5. At D14.5, ADAMDEC-1 amount was rapidly decreased.

In the miscarriage placentas collected from the pairs of CBA females and DBA males (CBA $\times$ DBA), ADAMDEC-1 expression level showed decreasing tendency as compared with normal sites (Fig. 1B). At D14.5, high expression of ADAMDEC-1 was detected at the miscarriage sites (Fig. 1C), however statistical differentiation was not detected because of the high deviation value, which indicated that ADAMDEC-1 expression was unstable in each miscarriage site. Associated with these results, the miscarriage sites from D14.5 showed dispersibility for placental and fetus degeneration, e.g., variations of discoloration degree and shrinkage sizes (Supplementary Fig. 1D).

\section{Localization of ADAMDEC-1 in pregnant mice}

Immunohistochemical analysis for ADAMDEC-1 revealed positive reaction at the extraembryonic ectoderm in the D6.5 egg cylinder (Fig. 1D). Diffuse localization was also detected at the most cells in the decidua basalis (Fig. 1E). At D10.5, strong reactivity for ADAMDEC-1 was found on UNK cells and their cytoplasmic granules (Fig. 1F). Positive uNK cells were frequently found to be surround and sometimes inside of the spiral arterioles at decidua basalis region. At D14.5, weak or moderate immunoreaction were seen on the trophoblasts at the placental labyrinth region especially on the cell membrane (Fig. $1 \mathrm{G})$. In the matured mouse placenta, elongated and thinned labyrinthine trophoblasts consist the maternal blood sinuses bearing cellular barrier for maternal and fetal substance exchange.

Fetal nuclear erythrocytes in normal placenta also showed strong or moderate positive reaction in the cytoplasm (Supplementary Fig. 2A). ADAMDEC-1 immunoreactions in fetal erythrocytes were found at D10.5, 12.5, and 14.5 of pregnancy and among them, anuclear matured-type erythrocytes in the fetal and maternal placental vessels showed negative reaction. In the fetal liver, a number of hematogenic cells showed ADAMDEC-1 positive reactions (Supplementary Fig. 2B). In the miscarriage placentas collected from DBA $\times$ CBA pairs, immunoreactions for ADAMDEC-1 were found at labyrinthine trophoblasts and the positive intensity and frequency were similar to those in the normal placentas (data not shown). On the other hand, uNK cells and fetal erythrocytes did not exist in the miscarriage placentas. 

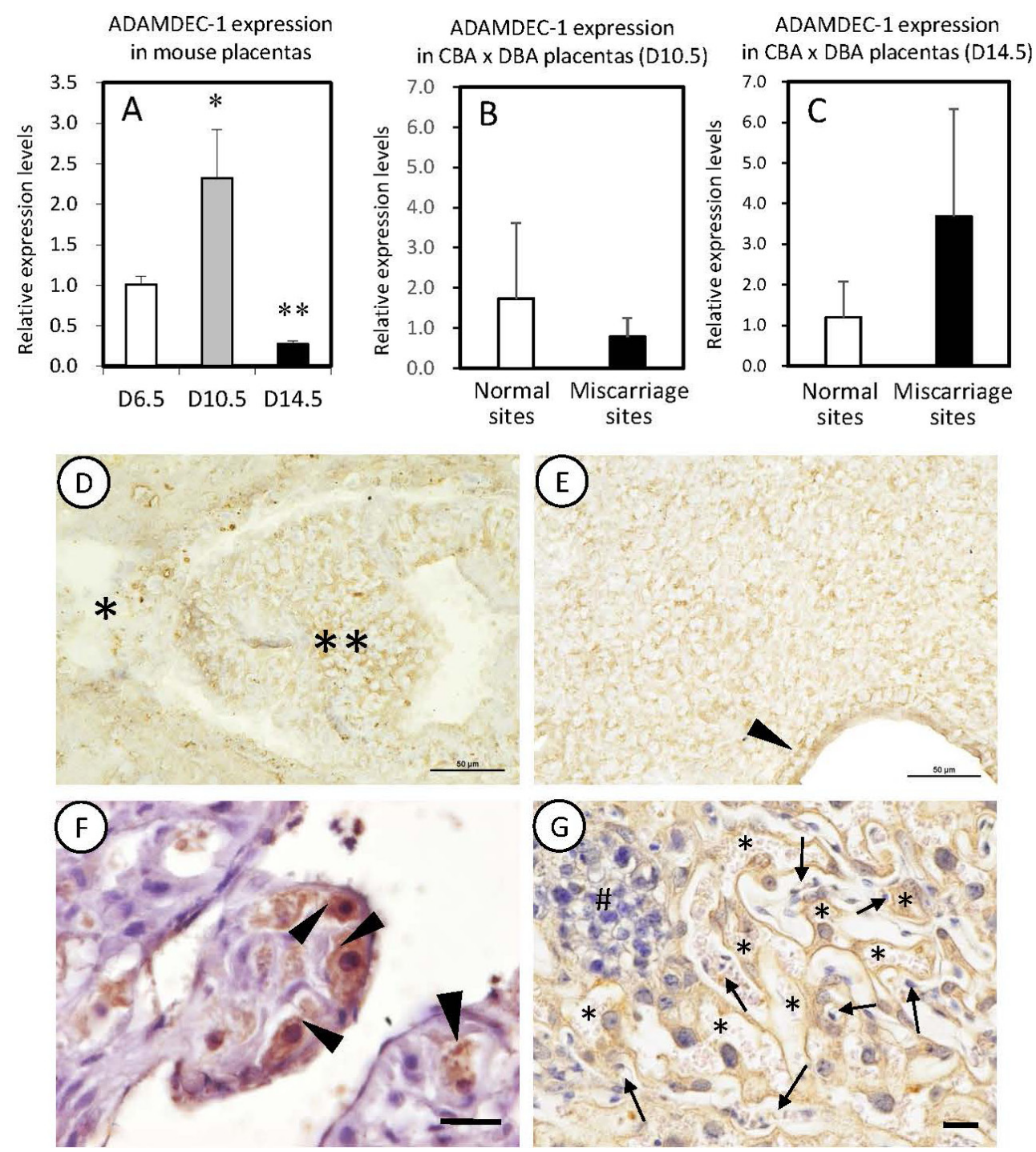

Fig. 1. A: qPCR analyses of a family of disintegrin and metalloproteinase (ADAMDEC)-1 expression in mouse normal placentas at the days 6.5, 10.5 and 14.5 of pregnancy (D6.5, D10.5, D14.5, respectively). ${ }^{*} P<0.05, * * P<0.01$ vs. D6.5. B, C: Comparison of ADAMDEC-1 expression between normal and miscarriage placentas collected from CBA/J mice mated with DBA $/ 2$ mice $(\mathrm{CBA} \times \mathrm{DBA})$. Statistical differences were not detected. D-G: Immunohistochemistry of ADAMDEC-1. At D6.5, positive reactions are seen in the cytoplasm of extraembryonic ectoderm $(\mathrm{D}, * *)$, but the area of ectoplacental cone is negative $(*)$. Positive reactions are also seen at most cells in the decidua basalis (E, D6.5) and the luminal epithelium (arrowheads). At D10.5, many uterine natural killer (uNK) cells in the decidua basalis region show positive reaction in the cytoplasm (F) and the granules (arrowheads). At D14.5, immunoreaction can be seen on the cell membrane of trophoblasts in the placental labyrinth region (G). Positive-labyrinthine trophoblasts form boundary walls between the maternal blood sinuses (asterisks) and fetal capillaries (arrows). Aggregation of the spongiotrophoblasts shows negative (\#). Bar=50 $\mu \mathrm{m}(\mathrm{D}, \mathrm{E})$ and $20 \mu \mathrm{m}(\mathrm{F}, \mathrm{G})$.

\section{ADAMDEC-1 effects on endothelial cells and vessels}

From the previous report [4] and results of immunohistochemical analyses, vascular effects were focused to evaluate ADAMDEC-1 functions. Administration of recombinant ADAMDEC-1 on the HUEhT-1 endothelial cells enhanced elongation of cell length and dilatation of the lumen structure (Fig. 2A-C). But the proliferation ability was decreased following ADAMDEC-1 treatment (Fig. 2D).

In the transplants of the VEGF-containing Matrigel, new capillaries were extending from the host mouse vessels (Fig. 3B and $3 C)$. Additional supplement of ADAMDEC-1 seemed to elevate the numbers of vessels in Matrigel and expanded lumen sizes of the host mouse vessels (Fig. 3D and 3E), whereas statistical differences were not detected.

Recombinant ADAMDEC-1 treatments to the pregnant mice did not show obvious influences on the numbers of normal and miscarriage sites, miscarriage rates, fetal weight, and placental weight (Supplementary Table 3). Also, ADAMDEC-1 treatments did not clearly induce blood coagulation, thrombus formation, and tissue degeneration in placentas caused in CBA x DBA miscarriage model. Morphometric measurements revealed that ADAMDEC-1 administration induced to enlarge the lumen size of fetal capillaries in placental labyrinth zone (Fig. 4A and 4B). Fetal capillaries near the maternal (decidua) side can contact directly with 

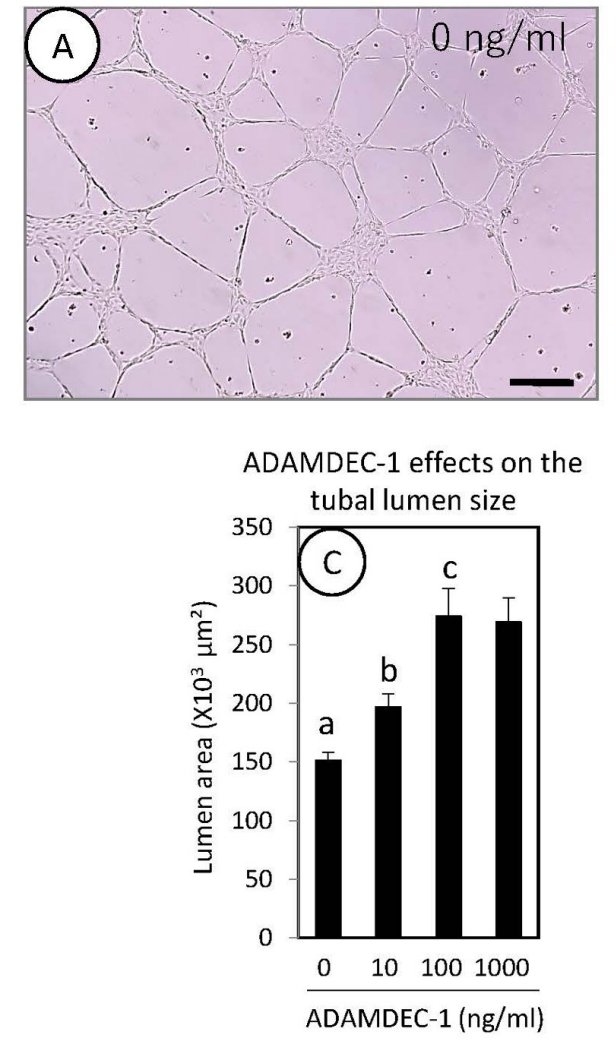

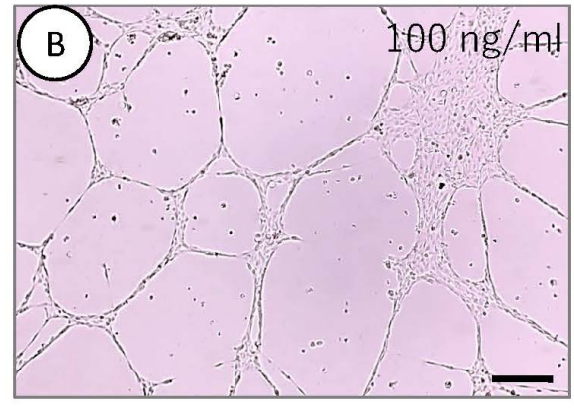

ADAMDEC-1 effects on the cell growth

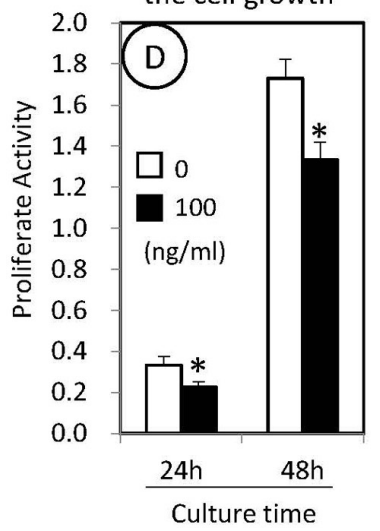

Fig. 2. Tubular formation by HUEhT-1 cells induced by culture on the Matrigel coated dishes (A, B). Scale bar=200 $\mu$ m. Recombinant a family of disintegrin and metalloproteinase (ADAMDEC)-1 effected to enlarge the area of tubal lumen on the concentration-dependent manner (C). Cell Counting Kit-8 (CCK8) mitochondria assay showed decrease of the proliferation activity after $100 \mathrm{ng} / \mathrm{ml}$ ADAMDEC-1 treatment (D, black column). Significant value; $P<0.05$, among the different marks (C) and $* P<0.05 \mathrm{vs.} 0 \mathrm{ng} / \mathrm{ml}$ (D). Data were represented as Mean \pm SE.
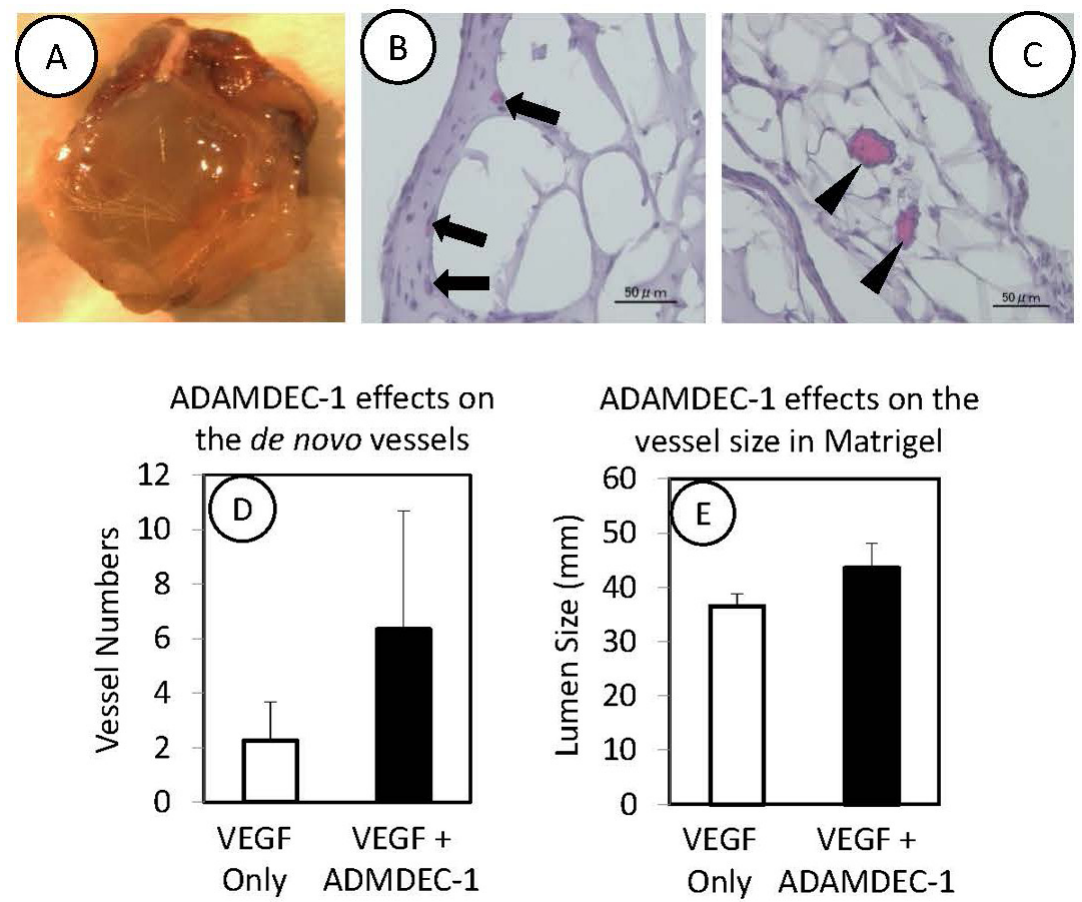

ADAMDEC-1 effects on the

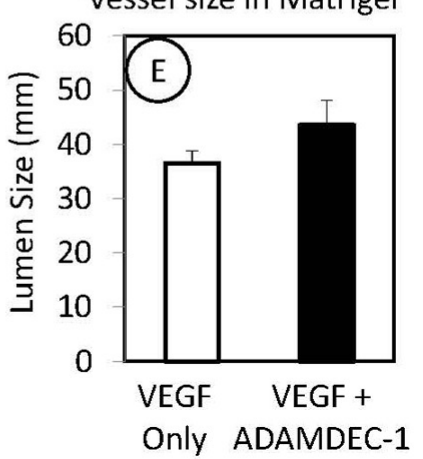

Fig. 3. A: Matrigel matrix plug collected from mouse axillary cavity. Cryostat section of Matrigel plug includes de novo vessels (B, arrows), extended from the recipient vessels distributing at the exterior mesenchymal tissue (C, arrowheads). A family of disintegrin and metalloproteinase (ADAMDEC)-1 treatment showed increase effects on the new vessels number in Matrigel (D) and the lumen size of the recipient vessels (E). $P=0.29$ (D) and 0.09 (E). 

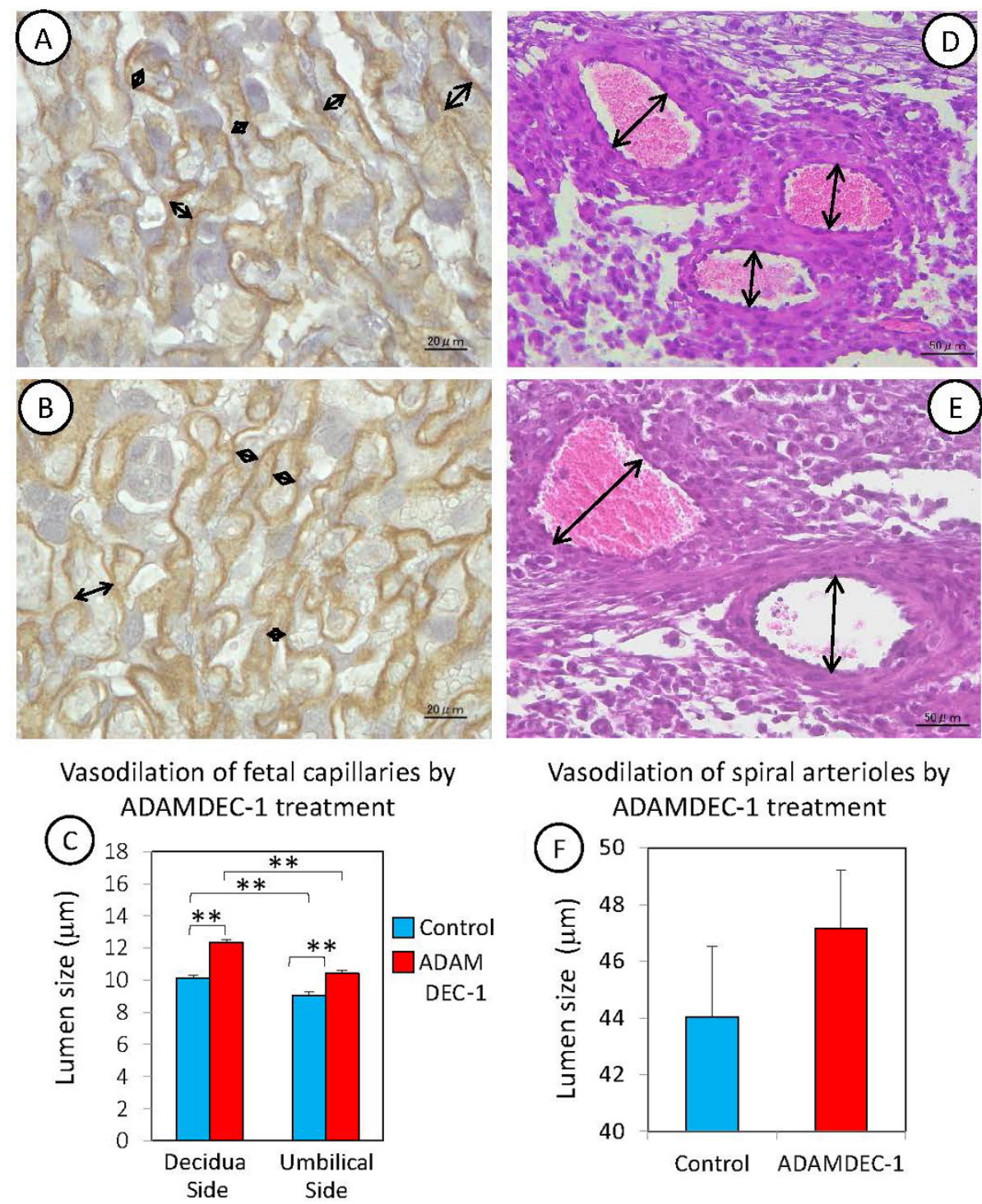

Vasodilation of spiral arterioles by ADAMDEC-1 treatment

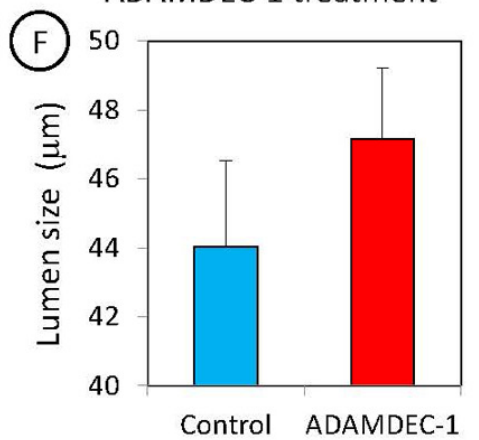

Fig. 4. Influences of a family of disintegrin and metalloproteinase (ADAMDEC)-1 administration to pregnant mice on D14.5. A, B: Bandeirasea simplicifolia Isolectin (BSI)-B4 immunostaining shows clear immunopositive capillary endothelium in the placental labyrinth regions at control (A) and ADAMDEC-1 treated mouse (B). Arrows indicate several examples for the measurement of lumen size and the results were compared distinctively at near the decidua (maternal side) and the umbilical (fetal side) areas, respectively $(\mathrm{C}, * * P<0.01)$. D, E: hematoxylineosin staining in the decidua basalis region. Lumen sizes of spiral arterioles were compared between control and ADAMDEC-1 treated mouse (arrows in D and E, respectively). Statistical evaluation shows dilating tendency of the vessels following ADAMDEC-1 administration (F, $P=0.15)$. Bar $=50 \mu \mathrm{m}(\mathrm{A}, \mathrm{B})$ and $20 \mu \mathrm{m}(\mathrm{D}, \mathrm{E})$.

the maternal blood including affluent oxygen and nutrient and send them to umbilical side capillaries. ADAMDEC-1 vasodilation effects are shown on both sides of capillaries (Fig. 4C). Spiral arterioles at the decidua basalis regions, carrying essential maternal nutrient to placenta, showed the tendency to enlarge the lumen size by ADAMDEC-1 treatment (Fig. 4D and 4E). But significant difference could not be detected (Fig. 4F).

\section{Other notable effects from ADAMDEC-1 administration}

ADAMDEC-1 administration increased appearance numbers of DBA lectin-positive uNK cells in decidua basalis region (Fig. $5 \mathrm{~A}$ and $5 \mathrm{~B}$ ). uNK cell recruitment was remarkable especially on the mature type of cells (criteria III) and distribution sites were notable around and inside of the spiral arterioles (Fig. 5C).

qPCR analyses were performed to confirm ADAMDEC-1 effects that can be supposed from the functions of ADAM families, concerning Notch signaling, disintegrin, and actin polymerization [6]. ADAMDEC-1 administration fluctuated gene expression degrees in placentas (Fig. 6). Especially, actinin $\alpha 1, \alpha 4$, and vinculin, relating to integrin-mediated cellular stabilization and formation of intracellular stress fibers, showed reduction following ADAMDEC-1 treatment. However, statistical differences were not detected in each analysis. 


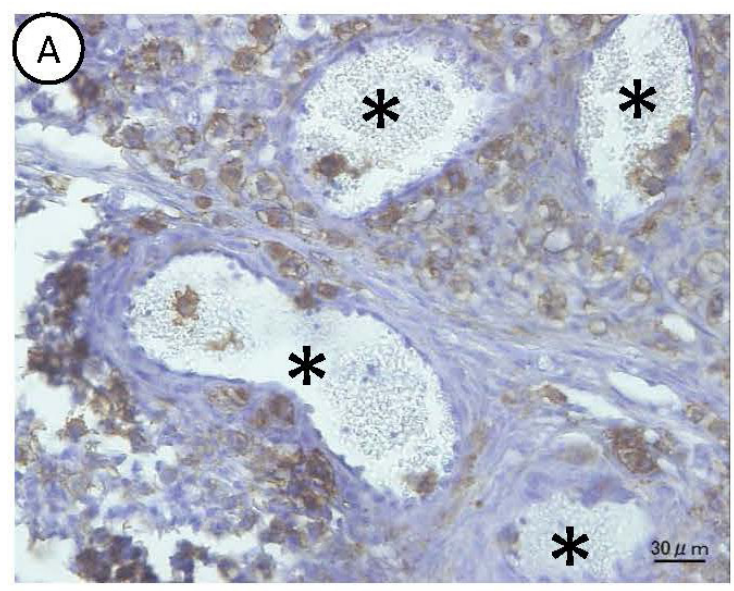

Cell densities and differential classification for uNK cells in the decidua basalis

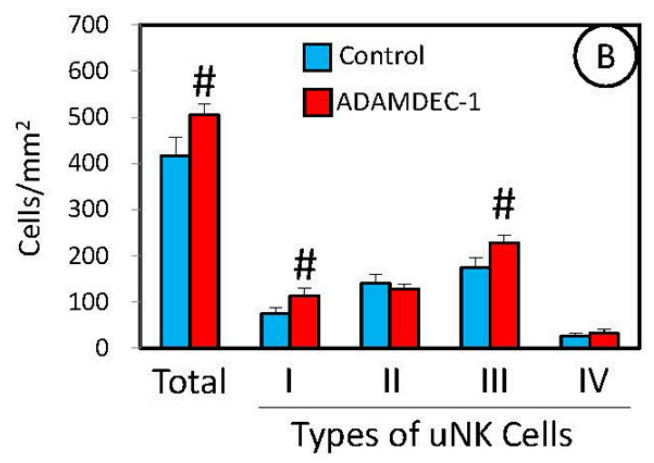

Cell densities and differential classification for uNK cells around and

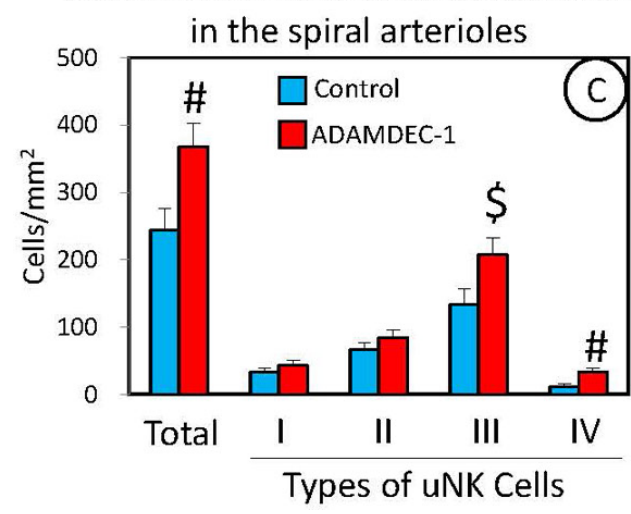

Fig. 5. A: Dolichos Biflorus Agglutinin (DBA)-lectin histochemical staining at D14.5 of the decidua basalis region following a family of disintegrin and metalloproteinase (ADAMDEC)-1 treatment. Abundant numbers of DBA lectin-positive uterine natural killer (uNK) cells can be observed, especially surround and inside of the spiral arterioles $(*)$. B, C: DBA lectin-positive uNK cells were classified by the morphological criteria (Paffaro et al., 2003 [23]). Cell densities were calculated in the whole region of decidua basalis (B) and at the circumscribed and inside areas of the spiral arterioles (C). ${ }^{\#} P<0.05,{ }^{\$} P<0.01$; vs. Control.
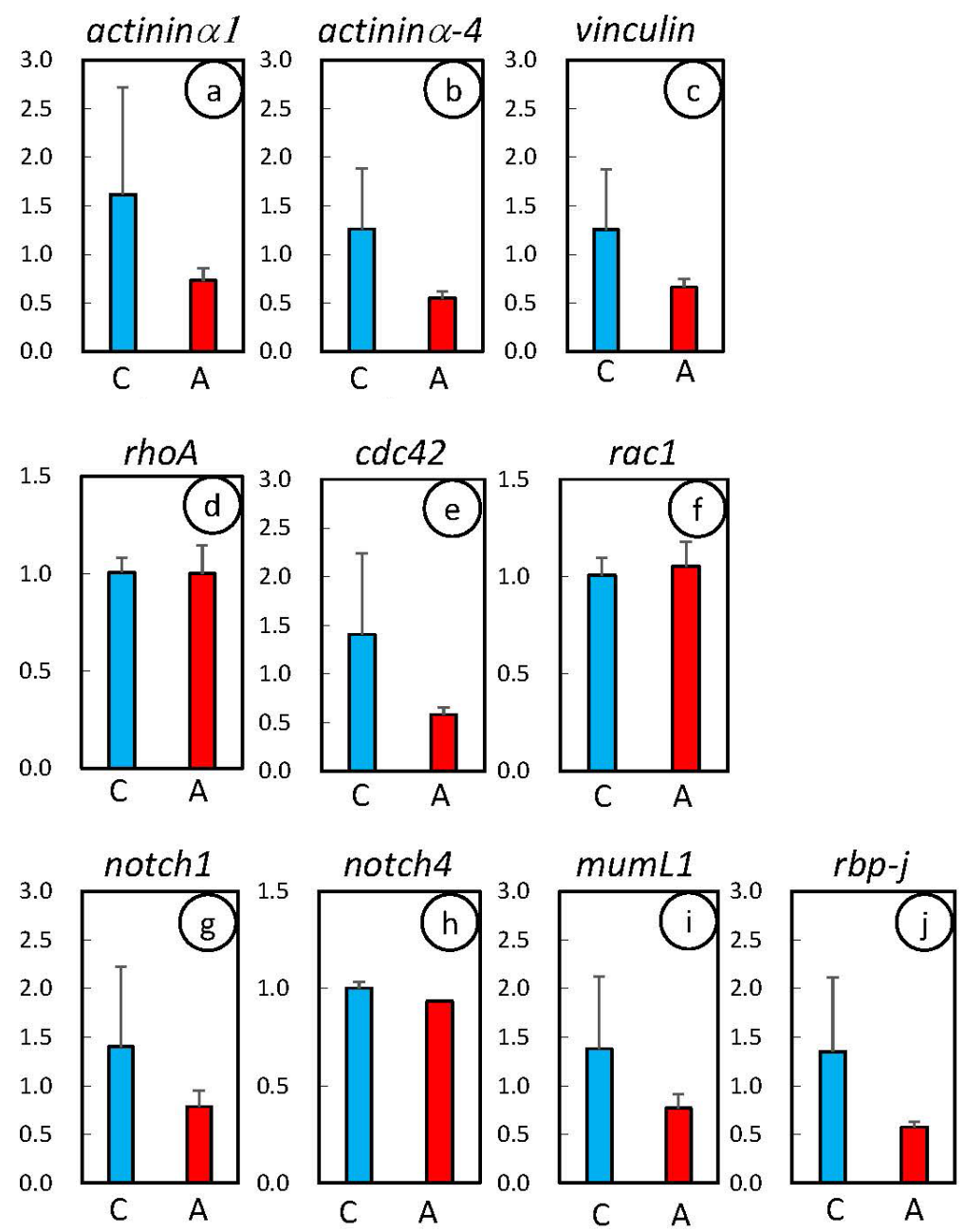

Fig. 6. a-j: Quantitative real-time RT-PCR analysis on the mRNA purified from D14.5 placenta following control (C, blue columns) and a family of disintegrin and metalloproteinase (ADAMDEC)-1 (A, red columns) treatments. Specific primers were used to detect actinin $\alpha 1$ (a), actinin $\alpha 4$ (b), vinculin (c), rhoA (d), $c d c 42$ (e), racl (f), notchl (g), notch4 (h), mumL1 (i), and $r b p-j$ (j). Y axis represents degrees for each molecular expression following the correction measures by gapdh expression and the statistical processing for relative expression intensity. 


\section{DISCUSSION}

Present study denied our previous hypothesis that is, ADAMDEC-1 may be involved in the induction process for spontaneous miscarriage [16]. qPCR analysis indicated disagreement of ADAMDEC-1 expression patterns with the periods for miscarriage initiation and advancement. Especially at D10.5, ADAMDEC-1 was decreased in the miscarriage sites of CBA x DBA model. Our observation in the normal mouse placenta also showed various distribution for ADAMDEC-1 positive cells. It seemed that ADAMDEC-1 is equipped innately in mouse tissue and has biological potentials reflecting cellular activities during normal pregnancy and tissue recovering after causing of miscarriage.

At the normal mouse placentas of D10.5, ADAMDEC-1 distribution was found at uNK cells. uNK cells have been known to have a vital role for pregnancy success involving in placental vasculature modification that is essential process for normal placentation $[9,10]$. In the process for hemochorial-type placentation (rodents and primates), maternal spiral arteries are destroyed by the fetal infiltrative trophoblasts, and then expandable maternal blood sinuses are formed de novo by the syncytium trophoblasts. uNK cells and trophoblasts can secrete VEGF and prepare VEGF gradient situation in developing placenta, which initiate and navigate vascular extension during early pregnant stages [5]. We found that ADAMDEC-1 treatment strengthened VEGF effects to increase neovascular extension into the Matrigel plugs. Furthermore, ADAMDEC-1 treatment showed solitary effects to extend the length and lumen size of epithelial cells in vitro and in vivo treatment of ADAMDEC-1 enlarged lumen sizes of the placental vessels derived both from maternal and fetal vessels. These vasoactive effects may be accounted for direct effects on the endothelial cells or indirect upregulation for uNK cells on the local function and differentiation.

At D14.5, ADAMDEC-1-immunoreaction in uNK cells became weak. It may be due to functional downregulation of uNK cells, which cause apoptosis and decrease in number at late pregnant periods [15]. On the other hand, ADAMDEC-1 positive reaction was also seen at the extraembryonic ectoderm in early stages of placental formation (D6.5). During placental development, these cells may be continuously expressing ADAMDEC-1 at the stages for trophectoderm and labyrinthine trophoblast. It was suggested that ADAMDEC-1 released from these trophoblasts promote vascular development during placental formation or control placental blood flow to keep normal pregnancy and fetal survival.

Notch signaling pathway is known to be critical in neural cell development [7] and segmentation during the normal somite formation [8]. Catalytic effects of ADAM family proteins and its cofactor gamma-secretase can cleave the transmembrane domain of Notch, and this event induces nuclear transportation of Notch intracellular domain that acts as a gene expression regulator. These effects are generally called as "regulated intramembrane proteolysis (RIP)" or "ectodomain shedding", which mediates receptormediated gene expression or regulates ligand-receptor interaction [6]. However, specific substrates of the ADAMDEC-1 have been clarified yet and its RIP effects are unclear.

Deficient mice for both subtypes of Notch 1 and Notch 4 display insufficiency for placental vascular development and fetal arterial development [14]. Also, single enhancement of Notch 4 expression during pregnancy evokes development delay in fetuses and suppression of vascular formation [30]. We examined the molecular fluctuation of $m u m L 1$ and $r b p-j$, which are pivotal molecules to bind Notch intracellular domain and gene expression [21, 22]. However, we could not find clear evidence for of Notch signaling activation in the ADAMDEC-1 treated placentas and correlation of Notch signaling pathway with ADAMDEC-1mediated placental vasculogenesis.

ADAM family proteins have proline-rich intracellular domain, which can bind to Src homology (SH) 3 adaptor molecules that couple to tyrosine kinase receptor and promote activation in Ras superfamily monomeric GTPases [11]. Rho proteins are members for Ras superfamily and involved with actin polymerization and rearrangement of cytoplasmic filament. Studies with Src-gene transfected fibroblasts verified that ADAM12, 15, and 19 are binding partners for SH3 molecules especially in the process for rearrangement of actin filament and formation of podosome [1]. ADAM family proteins may be associated with intracellular stress fiber formation and enhancement of migration ability. We tried to find evidence of ADAMDEC-1 effects on gene expression for $\operatorname{rohA}, \mathrm{racl}$, and $c d c 42$ in placentas, which belong to the Rho family protein and essential for SH3 signal transduction [29]. However, sufficient signs for gene upregulation operated by ADAMDEC-1 treatment could not be detected.

On the other hand, ADAMDEC-1 effected to downregulate placental gene expression for vinculin and actinin subfamilies. These proteins distribute at the region of focal adhesion apparatus that connects cells on the extracellular matrix. Vinculin acts as intracellular attachment protein and interlock between cytoplasmic stress fibers and membranous integrin [3]. $\alpha$-Actinins can bundle actin filaments and allow myosin II interaction to promote stress fiber formation [28]. ADAM family proteins are known to have "disintegrin domain" at the extracellular region, which can discriminate RGD sequences in the integrin molecule and interfere specific biding to the fibronectin [6]. ADAMDEC-1 treatments might loosen integrin and extracellular connection and consequently enhanced plasticity of intracellular actin filaments and stress fibers. Cell shape alteration is accompanied with dissolution of stress fibers and dispensation of actin filaments to the ruffled borders [12]. ADAMDEC-1 may associate with cell elongation via actin filament rearrangement especially in epithelial cells and trophoblasts in mouse placenta.

ADAMTS-13, a member of ADAM superfamily, can promote proliferation and tubal formation in endothelial cell line [18]. ADAMTS-13 is also able to support angiogenic effects of VEGF by initiating phosphorylation for VEGF receptor-2 and acceleration of VEGF expression in endothelial cells [17]. Besides, ADAMTS-13 is proven to promote trophoblast proliferation, migration, extension with two-dimensional cellular connection, suggesting close association with embryo implantation, placental formation, and tissue regeneration in pregnant uterus [31]. On the other hand, another ADAM family protein such as ADAMTS-7 downregulated cell activities in trophoblasts [19] and emphasized a pathogenicity correlation with preeclampsia [32]. Our and recent studies indicated ambivalent functionality among ADAM family proteins and/or much room for experimental evaluation 
towards breakthrough of ADAM family in reproductive contribution.

Our findings of ADAMDEC-1 on hematopoietic cells in placenta and fetal liver may indicate another aspect for metalloproteinase on biological contribution. Matrix metalloproteinase (MMP)-9 exerts "RIP" effect to cleave and release membrane-bounded kit ligand that promotes differentiation and reconstruction of stem/progenitor cells from vascular niche in bone marrow [13]. Kit ligand is also called as "stem cell factor" and can induce hematopoiesis. Likewise, membrane-type 1 matrix metalloproteinase (MT1-MMP) exerts hematopoietic effects associated with hypoxia-inducible factor (HIF)-1 [20]. HIF is known as transcriptional activator on major hematopoietic factors, such as erythropoietin, VEGF, and kit ligand. MT1-MMP is proven to downregulate the factor inhibiting HIF-1 (FIH-1) and to maintain HIF-1 expression level in the stromal niche cells [20]. The MMP domain in ADAMDEC-1 in placenta and fetal liver may possess similar hematopoietic function and become a sign for normal outcomes on the process of fetal development.

Present study presented functional participation of ADAMDEC-1 in mouse pregnant uteri. ADAMDEC-1 expression was not fully corresponded with miscarriage initiation timing but rather with early placental formation periods. It was suggested that ADAMDEC-1 is related to vascular kinetics in developing placentas such as vascular dilation of lumen size, which may be collaborated with VEGF. Effects on the endothelium and uNK cells were also suggested to promote cellular extension and migration that may be accounted for actin filament modification. We found just a hint for biological roles, and it seemed that ADAMDEC-1 and ADAM family are promising for understanding of cytological alteration and biological events in mammal placentation process.

POTENTIAL CONFLICTS OF INTEREST. The authors have nothing to disclose.

ACKNOWLEDGMENT. This work was supported by JSPS KAKENHI, Grant No. 26450445 (to K-T.K.).

\section{REFERENCES}

1. Abram, C. L., Seals, D. F., Pass, I., Salinsky, D., Maurer, L., Roth, T. M. and Courtneidge, S. A. 2003. The adaptor protein fish associates with members of the ADAMs family and localizes to podosomes of Src-transformed cells. J. Biol. Chem. 278: 16844-16851. [Medline] [CrossRef]

2. Anno, K., Hayashi, A., Takahashi, T., Mitsui, Y., Ide, T. and Tahara, H. 2007. Telomerase activation induces elongation of the telomeric singlestranded overhang, but does not prevent chromosome aberrations in human vascular endothelial cells. Biochem. Biophys. Res. Commun. 353: 926-932. [Medline] [CrossRef]

3. Axelrod, J., Birchmeier, W., Burridge, K., Couchman, J., Damsky, C., Falk, M., Garrod, D., Goodenough, D., Humphries, M., Hynes, R., Keegstra, K., Sheng, M., Streuli, C., Takeichi, M. and Yamada, K. 2008. Integrins are transmembrane heterodimers that link to the cytoskeleton. p. 1170. In: Molecular Biology of the Cell, fifth ed. (Albert, B., Johnson, A., Lewis, J., Raff, M., Roberts, K. and Walter, P. eds.), Garland Science, New York.

4. Baran, N., Kelly, P. A. and Binart, N. 2003. Decysin, a new member of the metalloproteinase family, is regulated by prolactin and steroids during mouse pregnancy. Biol. Reprod. 68: 1787-1792. [Medline] [CrossRef]

5. Croy, B. A., van den Heuvel, M. J., Borzychowski, A. M. and Tayade, C. 2006. Uterine natural killer cells: a specialized differentiation regulated by ovarian hormones. Immunol. Rev. 214: 161-185. [Medline] [CrossRef]

6. Edwards, D. R., Handsley, M. M. and Pennington, C. J. 2008. The ADAM metalloproteinases. Mol. Aspects Med. 29: 258-289. [Medline] [CrossRef]

7. Gilbert, S. G. 2006. The notch pathway: Juxtaposed ligands and receptors. pp. 160-161. In: Developmental Biology, eighth ed., Sinauer Associates Inc., Sunderland.

8. Gilbert, S. G. 2006. Coordinating waves and clocks in somite formation. p. 448. In: Developmental Biology, eighth ed., Sinauer Associates Inc., Sunderland.

9. Guimond, M. J., Luross, J. A., Wang, B., Terhorst, C., Danial, S. and Croy, B. A. 1997. Absence of natural killer cells during murine pregnancy is associated with reproductive compromise in TgE26 mice. Biol. Reprod. 56: 169-179. [Medline] [CrossRef]

10. Guimond, M. J., Wang, B., Fujita, J., Terhorst, C. and Croy, B. A. 1996. Pregnancy-associated uterine granulated metrial gland cells in mutant and transgenic mice. Am. J. Reprod. Immunol. 35: 501-509. [Medline] [CrossRef]

11. Harberd, N., Bourne, H., Bray, D., Briscoe, J., Ferrel, J., Freeman, M., Gilman, A., Ghosh, S., Hall, A., Heldin, C.E., Irvine, R., Meyerowitz, E., Nusse, R., Pawson, T., Pitcher, J. and Stephens, L. 2008. Protein with SH2 domains bind to phosphorylated tyrosines. pp. 924-928. In: Molecular Biology of the Cell, fifth ed. (Albert, B., Johnson, A., Lewis, J., Raff, M., Roberts, K. and Walter, P. eds.), Garland Science, New York.

12. Hay, E. D. and Svoboda, K. K. 1989. Extracellular matrix interaction with the cytoskeleton. pp. 147-167. In: Cell shape determinants, regulation, and regulatory role (Wifred, D. S. and Bronner, F. eds.), Academic Press. San Diego.

13. Heissig, B., Hattori, K., Dias, S., Friedrich, M., Ferris, B., Hackett, N. R., Crystal, R. G., Besmer, P., Lyden, D., Moore, M. A. S., Werb, Z. and Rafii, S. 2002. Recruitment of stem and progenitor cells from the bone marrow niche requires MMP-9 mediated release of kit-ligand. Cell 109: 625-637. [Medline] [CrossRef]

14. Krebs, L. T., Xue, Y., Norton, C. R., Shutter, J. R., Maguire, M., Sundberg, J. P., Gallahan, D., Closson, V., Kitajewski, J., Callahan, R., Smith, G. H., Stark, K. L. and Gridley, T. 2000. Notch signaling is essential for vascular morphogenesis in mice. Genes Dev. 14: 1343-1352. [Medline]

15. Kusakabe, K., Okada, T., Sasaki, F. and Kiso, Y. 1999. Cell death of uterine natural killer cells in murine placenta during placentation and preterm periods. J. Vet. Med. Sci. 61: 1093-1100. [Medline] [CrossRef]

16. Kusakabe, K., Naka, M., Ito, Y., Eid, N. and Otsuki, Y. 2008. Regulation of natural-killer cell cytotoxicity and enhancement of complement factors in the spontaneously aborted mouse placenta. Fertil. Steril. 90 Suppl: 1451-1459. [Medline] [CrossRef]

17. Lee, M., Keener, J., Xiao, J., Long Zheng, X. and Rodgers, G. M. 2015. ADAMTS13 and its variants promote angiogenesis via upregulation of VEGF and VEGFR2. Cell. Mol. Life Sci. 72: 349-356. [Medline] [CrossRef]

18. Lee, M., Rodansky, E. S., Smith, J. K. and Rodgers, G. M. 2012. ADAMTS13 promotes angiogenesis and modulates VEGF-induced angiogenesis. Microvasc. Res. 84: 109-115. [Medline] [CrossRef]

19. Meng, Y. H., Zhang, J. B., Sun, Y. L. and Liu, X. L. 2020. ADATMS-7 regulates the focal adhesion kinase signaling and promotes invasiveness of 
trophoblasts in early pregnancy. Placenta 92: 54-61. [Medline] [CrossRef]

20. Nishida, C., Kusubata, K., Tashiro, Y., Gritli, I., Sato, A., Ohki-Koizumi, M., Morita, Y., Nagano, M., Sakamoto, T., Koshikawa, N., Kuchimaru, T., Kizaka-Kondoh, S., Seiki, M., Nakauchi, H., Heissig, B. and Hattori, K. 2012. MT1-MMP plays a critical role in hematopoiesis by regulating HIF-mediated chemokine/cytokine gene transcription within niche cells. Blood 119: 5405-5416. [Medline] [CrossRef]

21. Oyama, T., Harigaya, K., Muradil, A., Hozumi, K., Habu, S., Oguro, H., Iwama, A., Matsuno, K., Sakamoto, R., Sato, M., Yoshida, N. and Kitagawa, M. 2007. Mastermind-1 is required for Notch signal-dependent steps in lymphocyte development in vivo. Proc. Natl. Acad. Sci. USA 104: 9764-9769. [Medline] [CrossRef]

22. Oyama, T., Harigaya, K., Sasaki, N., Okamura, Y., Kokubo, H., Saga, Y., Hozumi, K., Suganami, A., Tamura, Y., Nagase, T., Koga, H., Nishimura, M., Sakamoto, R., Sato, M., Yoshida, N. and Kitagawa, M. 2011. Mastermind-like 1 (MamL1) and mastermind-like 3 (MamL3) are essential for Notch signaling in vivo. Development 138: 5235-5246. [Medline] [CrossRef]

23. Paffaro, V. A. Jr., Bizinotto, M. C., Joazeiro, P. P. and Yamada, A. T. 2003. Subset classification of mouse uterine natural killer cells by DBA lectin reactivity. Placenta 24: 479-488. [Medline] [CrossRef]

24. Papaspyridonos, M., Smith, A., Burnand, K. G., Taylor, P., Padayachee, S., Suckling, K. E., James, C. H., Greaves, D. R. and Patel, L. 2006. Novel candidate genes in unstable areas of human atherosclerotic plaques. Arterioscler. Thromb. Vasc. Biol. 26: 1837-1844. [Medline] [CrossRef]

25. Takeshita, A., Kondo, T., Okada, T. and Kusakabe, K. T. 2010. Elevation of adipsin, a complement activating factor, in the mouse placenta during spontaneous abortion. J. Reprod. Dev. 56: 508-514. [Medline] [CrossRef]

26. Takeshita, A., Kusakabe, K. T., Hiyama, M., Kuniyoshi, N., Kondo, T., Kano, K., Kiso, Y. and Okada, T. 2014. Dynamics and reproductive effects of complement factors in the spontaneous abortion model of CBA/J $\times$ DBA/2 mice. Immunobiology 219: 385-391. [Medline] [CrossRef]

27. Takeshita, A., Nagaishi, S., Kondo, T., Okada, T. and Kusakabe, K. T. 2010. Regulation of complement activity via the alternative pathway in placentas of mouse spontaneous abortions. J. Vet. Med. Sci. 72: 1375-1377. [Medline] [CrossRef]

28. Theriot, J., Bourne, H., Goldstein, L., Hall, A., Howard, J., Machesky, L., Mitchison, T. and Vale, R. 2008. Cross-linking proteins with distinct properties organize different assembles of actin filaments. pp. 1006-1008. In: Molecular Biology of the Cell, fifth ed. (Albert, B., Johnson, A., Lewis, J., Raff, M., Roberts, K. and Walter, P. eds.), Garland Science, New York.

29. Theriot, J., Bourne, H., Goldstein, L., Hall, A., Howard, J., Machesky, L., Mitchison, T. and Vale, R. 2008. Members of the Rho protein family cause major rearrangements of the actin cytoskeleton. pp. 1041-1043. In: Molecular Biology of the Cell, fifth ed. (Albert, B., Johnson, A., Lewis, J., Raff, M., Roberts, K., Walter, P. eds.), Garland Science, New York.

30. Uyttendaele, H., Ho, J., Rossant, J. and Kitajewski, J. 2001. Vascular patterning defects associated with expression of activated Notch4 in embryonic endothelium. Proc. Natl. Acad. Sci. USA 98: 5643-5648. [Medline] [CrossRef]

31. Xiao, J., Feng, Y., Li, X., Li, W., Fan, L., Liu, J., Zeng, X., Chen, K., Chen, X., Zhou, X., Zheng, X. L. and Chen, S. 2017. Expression of ADAMTS13 in normal and abnormal placentae and its potential role in angiogenesis and placenta development. Arterioscler. Thromb. Vasc. Biol. 37: 1748-1756. [Medline] [CrossRef]

32. Zhang, L., Zhao, F., Li, C., Li, H., Tang, Q., Chen, Y., Yao, Y., Ding, Z., Xu, Y., Chen, A. and Liu, S. 2020. Hypomethylation of DNA promoter upregulates ADAMTS7 and contributes to HTR-8/SVneo and JEG-3 cells abnormalities in pre-eclampsia. Placenta 93: 26-33. [Medline] [CrossRef] 